\title{
Mental health resources and awareness of anxiety and depressive disorders in general hospitals in China
}

\author{
SHISHENG HAO ${ }^{*}$ \\ LING XIAO ${ }^{*}$ \\ HUILING WANG ${ }^{2}$ \\ ZHONGCHUN LiU2 \\ CAI NAN2 \\ GAOHUA WANG 2 \\ https://orcid.org/0000-0003-4716-112X \\ 1 Institute of Neuropsychiatry, Renmin Hospital of Wuhan University, Wuchang, Wuhan, China \\ 2 Department of Psychiatry, Renmin Hospital of Wuhan University, Wuchang, Wuhan, China. \\ *These authors contributed equally to this study.
}

Received: 11/26/2018 - Accepted: 5/21/2019

DOl: 10.1590/0101-60830000000205

\begin{abstract}
Background: Mental health disorders are common in China. There is a lack of knowledge and resources of mental health in China. Objectives: To assess the levels of psychiatric resources and services in general hospitals in China. Methods: Data regarding psychiatric departments, wards and staff were collected from 57 general hospitals in four provinces of China (Hubei, Zhejiang, Heilongjiang and Yunnan) between April 2014 and June 2014. Questionnaires were distributed to 1,200 non-psychiatric clinicians. Results: Among the 57 hospitals, 50 provided mental health services, 36 had mental health wards, and seven had neither mental health clinics nor wards. The median number of mental health clinicians was six per hospital. The median number of specialized nurses was 42 per hospital. A total of 1,152 non-psychiatric clinicians with a career duration of $9.4 \pm 8.9$ years returned completed questionnaires. Only $6.9 \%$ reported a good understanding of the manifestation of anxiety and depressive disorders, $4.5 \%$ reported a good understanding of the diagnostic criteria, and $3.8 \%$ reported a good understanding of the treatment protocols. Discussion: There is inadequate awareness of anxiety and depressive disorders among non-psychiatric clinicians in general hospitals in China. This awareness/understanding increased with increasing hospital level.
\end{abstract}

Hao S et al. / Arch Clin Psychiatry. 2019;46(4):107-12

Keywords: Anxiety and depressive disorders, China, general hospitals, mental health resources.

\section{Introduction}

The World Health Organization has estimated that the prevalence of mental health disorders is $2.4 \%-18.2 \%$, based on data from 14 countries ${ }^{1}$. Mental health disorders are also common in China, with a reported prevalence of up to $17.5 \%^{2}$. The aim of modern mental health care is the restoration of mental health and social functions ${ }^{3}$. In addition to primary mental health disorders, secondary anxiety and depressive disorders can develop in patients with primary physiological diseases during the course of the disease and its treatment. A survey of patients with severe cardiovascular diseases in the USA revealed that $20.5 \%$ screened positive for depression and $18.5 \%$ screened positive for generalized anxiety disorder ${ }^{4}$. Comparable results have been obtained for patients with other diseases in various countries, including China ${ }^{5}$.

Many patients with mental health disorders first consult at general hospitals ${ }^{6}$. Since the signs and symptoms of mental health disorders may be very subtle ${ }^{3}$, the diagnosis and management of these disorders by clinicians in general hospitals might be suboptimal ${ }^{7}$. In Western countries, the incidence of anxiety and depressive disorders in patients in general hospitals is $8 \%-53.6 \%$, and it has been estimated that only $10 \%$ of cases are diagnosed and treated reasonably ${ }^{8}$. A large-scale clinical study conducted in Shenyang (China) in 2008 reported that the incidences of anxiety and depressive disorders in general hospital were $11 \%$ and $9.8 \%$, respectively, and that the rate of therapy was approximately $4 \% 9$. Another survey in Wuhan in 2010 revealed that the incidence of all forms of depression in general hospitals was $16 \%$ and that most patients were not diagnosed and treated efficiently ${ }^{10}$. A survey conducted in Shanghai in 2011 found that $13.4 \%$ of patients in general hospitals were diagnosed with mild depression and $18.5 \%$ were diagnosed with severe depression; of these, only $3.9 \%$ and $8.7 \%$, respectively, were treated appropriately ${ }^{11}$.

However, evidence from previous studies has indicted that mental health knowledge may be inadequate among healthcare workers in general hospitals. For example, a survey in India concluded that nurses in general hospitals had inadequate psychiatric knowledge and skills ${ }^{12}$. Moreover, when provided with vignettes describing a patient with depression, generalized anxiety disorder or schizophrenia, the disorder was misdiagnosed by more than $40 \%$ of non-mental health professionals working in general hospitals in China ${ }^{13}$. Similarly, the accuracy of the initial psychiatric diagnosis of inpatients in a hospital in Saudi Arabia was only 50\% for depression and $46 \%$ for anxiety disorders ${ }^{14}$. Studies such as these highlight the need for in-service training of clinicians and nurses in general hospitals to improve their knowledge of mental health care. Furthermore, it is generally considered that the number of psychiatric physicians in China is inadequate ${ }^{15}$, with some general hospitals having no psychiatry department. Such a lack of resources would also be expected to impact negatively on the diagnosis and treatment of psychiatric diseases ${ }^{16}$. 
Nevertheless, no large-scale survey has been conducted to examine the status of mental health care in non-psychiatric hospital departments in China. Therefore, the present study aimed to assess the level of psychiatric resources and services in general hospitals in China as well as the mental health-related training and knowledge base of non-psychiatric clinicians working in these hospitals. It was anticipated that this novel information would raise awareness of any deficiencies in mental health care provision in general hospitals in China and thereby facilitate the development and widespread implementation of new healthcare strategies to improve the diagnosis and treatment of patients with anxiety or depressive disorders.

\section{Methods}

\section{Study design and subjects}

A cross-sectional survey was conducted between April 2014 and June 2014 in 57 general hospitals located in four provinces: Hubei (central China), Zhejiang (eastern China), Heilongjiang (northern China) and Yunnan (southern China). It was anticipated that the selection of four provinces in different geographical regions would increase the likelihood that the findings would be generalizable to China as a whole. These general hospitals were selected by the cluster stratified random sampling method. Clinicians in each general hospital were selected by simple random sampling method at a rate of about $20 \%$. Primary, secondary and tertiary hospitals were included (in China, primary hospitals are typically affiliated with towns and contain less than 100 beds; secondary hospitals are typically affiliated with medium-sized cities/districts and contain 100-500 beds; and tertiary hospitals provide comprehensive services at a city/provincial/ national level and typically have more than 500 beds). Data about the distribution of patients in wards and outpatient clinics and allocation of human resources for mental health were extracted from hospital records. The survey was administered to a total of 5,987 clinicians working in 16 department types: cardiology, neurology, neurosurgery, emergency medicine, hematology, gastroenterology, oncology, obstetrics and gynecology, respiratory, endocrinology, nephrology, pediatrics, internal medicine, genitourinary surgery, digestive medicine and otolaryngology. Using strict confidentiality principles and voluntary participation, 1,200 of 5,987 nonpsychiatric/non-psychological physicians were invited to participate in the study.

This study was approved by the ethics committee of our hospital (No. 2012-031). All the respondents provided informed written consent for inclusion in the study and completed the survey.

\section{Data collection}

The following data were collected: characteristics of the psychiatry department (wards, outpatient clinic only or neither); number of nurses and number of clinicians in the psychiatry department; the professional grades of the clinicians and nurses in the psychiatry department (senior, intermediate or junior); education level of the clinicians and nurses (doctor degree or above, master degree, bachelor degree, or junior college degree or below); number of patients in the psychiatry department; and number of consultations in the psychiatry department.

\section{Questionnaire}

A questionnaire designed in-house was administered to 1,200 non-psychiatric/non-psychological clinicians in order to collect general information as well as self-reported data regarding mental health-related learning and training, knowledge about anxiety and depressive disorders, attitude with mental health provision in general hospitals and recommendations to improve mental health provision in general hospitals.
For information regarding mental health-related learning and training, the participant was asked whether or not they had learnt psychiatry, clinical psychology or health psychology before graduation, whether or not they had received training in psychotherapy after graduation, and whether or not they had attended relevant academic lectures. The respondents were asked to provide yes/no answers to these items in the questionnaire.

The self-evaluation of knowledge of anxiety and depressive disorders was divided into three dimensions (manifestation, diagnostic criteria and treatment) and evaluated using a 5-point scale, as follows: $5=$ good understanding with relevant professional diagnostic and treatment skills; $4=$ reasonable understanding with relevant professional knowledge but limited ability to diagnose and treat the disorders; 3 = basic theoretical understanding of psychiatry/ psychology with some formal professional training but no relevant clinical practice; 2 = little understanding of psychiatry/psychology and no relevant professional training but had obtained some relevant knowledge by attending seminars or through other means; $1=$ no understanding of psychiatry/psychology and no relevant training.

\section{Statistical analysis}

SPSS 19.0 (IBM Corp., Armonk, NY, USA) was used for the analysis. Continuous variables are presented as the mean \pm standard deviation (SD) or median (range). Categorical variables are presented as frequency (percentage), and compared using chi-square test. $P<$ 0.05 was considered statistically significant.

\section{Results}

\section{Psychiatric care resources in general hospitals}

A total of 57 hospitals were surveyed in this study, including 42 (73.7\%) tertiary hospitals, 13 (22.8\%) secondary hospitals, and two (3.5\%) primary hospitals. Among the 57 hospitals, $50(87.7 \%)$ provided mental health services and $36(63.2 \%)$ had mental health wards, while seven $(12.3 \%)$ had neither mental health clinics nor wards. Each hospital had 0-40 mental health clinicians, with a median of six per hospital and a total number of 381 . The number of psychiatric nurses ranged 0-147, with a median of 42 per hospital and a total number of 673 . At these 57 hospitals, mental health clinicians accounted for $2.2 \%$ of the total number of clinicians, and psychiatric nurses accounted for $4.6 \%$ of the total number of nurses. Information regarding the psychiatric medical staff is shown in Table 1.

During each of 2010, 2011 and 2012, the number of patients seen in these mental health clinics and wards was 10,468, 16,298 and 23,008, respectively, and the total number of consultations at the psychiatric departments was $2,684,4,841$ and 5,973, respectively (Table 1).

\section{Characteristics of the non-psychiatric clinicians surveyed by questionnaire}

A total of 1,200 distributed questionnaires were completed and returned by the non-psychiatric clinicians. Of these, 1,152 questionnaires were included in the final analysis $(96.0 \%$ participation rate), and the remaining 48 (4.0\%) were excluded due to missing information or non-standard answers. Among the 1,152 non-psychiatric clinicians, 468 (40.6\%), 435 (37.8\%) and 249 (21.6\%) were from tertiary, secondary and primary hospitals, respectively. The age, gender, education level, professional grade and professional experience of the respondents are shown in Table 2. These 1,152 clinicians originated from 16 different departments, including cardiology, neurology, neurosurgery, emergency medicine, hematology, gastroenterology, oncology, obstetrics and gynecology, respiratory, endocrinology, nephrology, pediatrics, internal medicine, genitourinary surgery, digestive medicine and otolaryngology. 
Table 1. Psychiatry department resources in 57 general hospitals in four provinces of China

\begin{tabular}{|c|c|}
\hline Characteristic & $\begin{array}{l}\text { General hospitals } \\
\quad(n=57)\end{array}$ \\
\hline \multicolumn{2}{|l|}{ Hospital level, n (\%) } \\
\hline Primary & 2 (3.5) \\
\hline Secondary & $13(22.8)$ \\
\hline Tertiary & $42(73.7)$ \\
\hline \multicolumn{2}{|l|}{ Psychiatry department, $\mathrm{n}(\%)$} \\
\hline Wards & $36(63.2)$ \\
\hline Outpatient clinics only & $14(24.5)$ \\
\hline No outpatient clinics or wards & $7(12.3)$ \\
\hline \multicolumn{2}{|l|}{ Medical staff in psychiatry departments, median (range) } \\
\hline Clinicians & $6(0-40)$ \\
\hline Nurses & $42(0-147)$ \\
\hline \multicolumn{2}{|l|}{$\begin{array}{l}\text { Professional grade of the } 381 \text { clinicians working in psychiatry } \\
\text { departments, } n(\%)\end{array}$} \\
\hline Senior & $96(25.2)$ \\
\hline Intermediate & $159(41.7)$ \\
\hline Junior & $126(33.1)$ \\
\hline \multicolumn{2}{|l|}{$\begin{array}{l}\text { Education level of the clinicians working in psychiatry } \\
\text { departments, } n(\%)\end{array}$} \\
\hline Doctor degree or above & $30(7.9)$ \\
\hline Master degree & $98(25.7)$ \\
\hline Bachelor degree & $238(62.5)$ \\
\hline Junior college degree or below & $15(3.9)$ \\
\hline \multicolumn{2}{|l|}{$\begin{array}{l}\text { Professional grade of the } 673 \text { nurses working in psychiatry } \\
\text { departments, } \mathrm{n}(\%)\end{array}$} \\
\hline Senior & $21(3.1)$ \\
\hline Intermediate & $161(23.9)$ \\
\hline Junior & $491(73.0)$ \\
\hline \multicolumn{2}{|l|}{$\begin{array}{l}\text { Education level of the nurses working in psychiatry } \\
\text { departments, } n(\%)\end{array}$} \\
\hline Doctor degree or above & $0(0)$ \\
\hline Master degree & $3(0.4)$ \\
\hline Bachelor degree or below & $670(99.6)$ \\
\hline \multicolumn{2}{|l|}{ Number of patients seen in the psychiatry departments, $n(\%)$} \\
\hline 2010 & $10,468(21.0)$ \\
\hline 2011 & $16,298(32.8)$ \\
\hline 2012 & $23,008(46.2)$ \\
\hline \multicolumn{2}{|l|}{ Number of psychiatric consultations, $\mathrm{n}(\%)$} \\
\hline 2010 & $2,684(19.9)$ \\
\hline 2011 & $4,841(35.9)$ \\
\hline 2012 & $5,973(44.2)$ \\
\hline
\end{tabular}

\section{Mental health-related learning and training of the 1152 non- psychiatric clinicians (self-reported)}

Among the respondents, 596 clinicians (51.7\%) had learnt psychiatry before graduation, $562(48.8 \%)$ had learnt clinical psychology before graduation, 230 (20.0\%) had learnt health psychology before graduation, 59 (5.1\%) had attended psychotherapy training after graduation, and $255(22.1 \%)$ had attended relevant academic lectures beyond working hours (Table 3 ).

\section{Knowledge of anxiety and depressive disorders of the 1,152 non-psychiatric clinicians (self-reported)}

Only $80(6.9 \%)$ clinicians considered that they possessed a good understanding of the manifestations of anxiety and depressive
Table 2. Characteristics of 1,152 non-psychiatry clinicians working in general hospitals in four provinces of China

\begin{tabular}{|l|c|}
\hline Characteristic & $\begin{array}{c}\text { Non-psychiatry clinicians } \\
(\mathrm{n}=1,152)\end{array}$ \\
\hline Gender, $\mathrm{n}(\%)$ & $665(57.7)$ \\
\hline Male & $487(42.3)$ \\
\hline Female & $32.2 \pm 8.4$ \\
\hline Age (years), mean \pm SD & $9.4 \pm 8.8$ \\
\hline Professional experience (years), mean \pm SD & \\
\hline Hospital level, $\mathrm{n}(\%)$ & $468(40.6)$ \\
\hline Tertiary & $435(37.8)$ \\
\hline Secondary & $249(21.6)$ \\
\hline Primary & $273(23.7)$ \\
\hline Professional grade, $\mathrm{n}(\%)$ & $600(52.1)$ \\
\hline Senior & $279(24.2)$ \\
\hline Intermediate & \\
\hline Junior & $85(7.4)$ \\
\hline Education level, $\mathrm{n}(\%)$ & $252(21.9)$ \\
\hline Doctor degree or above & $484(42.0)$ \\
\hline Master degree & $331(28.7)$ \\
\hline Bachelor degree & \\
\hline Junior college degree or below & \\
\hline
\end{tabular}

disorders, $52(4.5 \%)$ clinicians reported that they had a good understanding of the diagnostic criteria, and 44 (3.8\%) clinicians believed that they had a good appreciation of the treatment protocols (Table 4). Notably, around $30 \%$ considered that they had little or no understanding of the manifestations of anxiety and depressive disorders, while around 50\% reported that they had little or no understanding of the diagnostic criteria or treatment protocols for these mental health disorders (Table 4). Non-psychiatry clinicians in tertiary hospitals had better understanding of the manifestations, diagnostic criteria, and treatment than those in primary and secondary hospitals (all $P<0.001$ ).

\section{Attitude with the mental health care system reported by the 1,152 non-psychiatric clinicians}

Assessment of the training programs needed indicated that all hospitals considered it necessary to provide relevant training to enhance knowledge and understanding of mental health care issues among medical staff, improve the consultation rates of psychiatric departments in general hospitals, and promote relevant research activities. Assessment of the desire of medical staff for mental health knowledge revealed strong interests and demands. The approaches used most commonly to obtain relevant mental health knowledge were ordered as: professional seminars, written and digital training materials, and academic conferences.

Assessment of attitude with the mental health system showed that $13.5 \%$ ( 156 respondents) of the clinicians were satisfied whereas $23.6 \%$ ( 272 respondents) were not. The vast majority of respondents considered it necessary to set up specialty clinics (86.4\%), set up specialized wards $(99.0 \%)$, conduct specialized training $(96.4 \%)$, improve scientific research (97.5\%), provide health-related education (97.2\%) and improve the rate of psychiatric consultations (98.3\%).

\section{Discussion}

The present study investigated 1,152 physicians in 57 general hospitals, and the results showed a lack of mental health resources in general hospitals in China. Among the 57 hospitals, there were mental health clinics in 50 hospitals (87.7\%), and mental health wards were provided by only 36 hospitals $(63.2 \%)$. The total numbers of patients seen in hospitals with mental health clinics and/or wards in 
Table 3. Mental health-related training of 1,152 non-psychiatry clinicians working in general hospitals in four provinces in China

\begin{tabular}{|l|c|c|c|c|c|}
\hline Type of training undertaken, $\mathrm{n}(\%)$ & $\begin{array}{c}\text { Total } \\
(\mathrm{n}=1,152)\end{array}$ & $\begin{array}{c}\text { Primary hospital } \\
(\mathrm{n}=249)\end{array}$ & $\begin{array}{c}\text { Secondary hospital } \\
(\mathrm{n}=435)\end{array}$ & $\begin{array}{c}\text { Tertiary hospital } \\
(\mathrm{n}=468)\end{array}$ & \begin{tabular}{l}
$P$ \\
\hline
\end{tabular} \\
\hline Learning before graduation & $596(51.7)$ & $28(11.2)$ & $107(24.6)$ & $461(98.5)$ & $<0.001$ \\
\hline Psychiatry & $562(48.8)$ & $14(5.6)$ & $87(20.0)$ & $461(98.5)$ & $<0.001$ \\
\hline Clinical psychology & $230(20.0)$ & $4(1.6)$ & $39(9.0)$ & $187(40.0)$ & $<0.001$ \\
\hline Health psychology & $59(5.1)$ & 0 & $6(1.4)$ & $53(11.3)$ & $<0.001$ \\
\hline Training in psychotherapy after graduation & $255(22.1)$ & $17(6.8)$ & $63(14.5)$ & $175(37.4)$ & $<0.001$ \\
\hline Attending relevant academic lectures & & & &
\end{tabular}

Table 4. Knowledge of anxiety and depressive disorders of 1152 non-psychiatry clinicians working in general hospitals in four provinces in China

\begin{tabular}{|c|c|c|c|c|c|}
\hline Score, $n(\%)$ & $\begin{array}{c}\text { Total } \\
(\mathrm{n}=1,152)\end{array}$ & $\begin{array}{l}\text { Primary hospital } \\
(\mathrm{n}=249)\end{array}$ & $\begin{array}{l}\text { Secondary hospital } \\
\qquad(\mathrm{n}=435)\end{array}$ & $\begin{array}{l}\text { Tertiary hospital } \\
(n=468)\end{array}$ & $P$ \\
\hline Manifestationabc & & & & & $<0.001$ \\
\hline 1 & $32(2.8)$ & $14(5.6)$ & $11(2.5)$ & $7(15.0)$ & \\
\hline 2 & $312(27.1)$ & 74 (29.7) & $193(44.4)$ & 45 (10.3) & \\
\hline 3 & $257(22.3)$ & 79 (31.7) & $93(21.4)$ & 85 (18.2) & \\
\hline 4 & 471 (40.91) & 67 (26.9) & $110(25.3)$ & $294(62.8)$ & \\
\hline 5 & $80(6.9)$ & $15(6.0)$ & $28(6.4)$ & $37(7.9)$ & \\
\hline Diagnostic criteriaabc & & & & & $<0.001$ \\
\hline 1 & $174(15.1)$ & 76 (30.5) & $62(14.3)$ & $36(7.7)$ & \\
\hline 2 & 401 (34.8) & $59(23.7)$ & $156(35.9)$ & $186(39.7)$ & \\
\hline 3 & $233(20.2)$ & 57 (22.9) & $92(21.1)$ & 84 (17.9) & \\
\hline 4 & $292(25.3)$ & $51(20.5)$ & $107(24.6)$ & $134(28.6)$ & \\
\hline 5 & $52(4.5)$ & $6(2.4)$ & $18(4.1)$ & $28(6.0)$ & \\
\hline Treatmentabc & & & & & $<0.001$ \\
\hline 1 & $186(16.1)$ & 79 (31.7) & 66 (15.2) & $41(8.8)$ & \\
\hline 2 & 436 (37.8) & 46 (18.5) & $203(46.7)$ & $187(40.0)$ & \\
\hline 3 & $219(19.0)$ & 73 (29.3) & $83(19.1)$ & 63 (13.5) & \\
\hline 4 & $267(23.2)$ & 49 (19.7) & 65 (14.9) & $153(32.7)$ & \\
\hline 5 & $44(3.8)$ & $2(0.8)$ & $18(4.1)$ & $24(5.1)$ & \\
\hline
\end{tabular}

a $P<0.001$, secondary hospital vs. primary hospital.

${ }_{b} P<0.001$, tertiary hospital vs. primary hospital.

c $P<0.001$, tertiary hospital vs. secondary hospital.

2010, 2011 and 2012 were 10,468, 16,298 and 23,008, respectively, presenting an increasing trend. Among the 1,152 clinicians surveyed, only $51.7 \%$ had learnt psychiatry, $48.8 \%$ had learnt clinical psychology, 20.0\% had learnt health psychology, 5.1\% had undertaken psychotherapy training, and $22.1 \%$ had attended relevant academic lectures. Notably, only $6.9 \%$ of clinicians considered that they possessed a good understanding of the manifestations of anxiety and depressive disorders, only $4.5 \%$ thought they had a good appreciation of the relevant diagnostic criteria and only $3.8 \%$ believed they had a good understanding of how to treat these disorders. Understanding increased with increasing hospital level. From the perspective of allocation of human resources, the available evidence suggests that China is inferior to Western countries with regard to the provision of mental health care in general hospitals ${ }^{15}$. Indeed, the mental health workforce is $5.16 / 100,000$ population in China, compared with $28.15 / 100,000$ population in upper-middle-income countries, $62.28 / 100,000$ population in high income countries, and $10.05 / 100,000$ population in the world (including China) ${ }^{15}$.

The awareness of mental illness has been rapidly increasing in the Chinese population ${ }^{17}$. In the present study, 2012 accounted for $46.2 \%$ of the total number of outpatients in the 3 years covered (2010-2012), and the number of consultations for mental illness reached $44.3 \%$, suggesting that the workload on psychiatric departments in China is increasing ${ }^{18}$. Nevertheless, a previous study in China showed that physicians in non-psychiatric departments only recognized about $27 \%$ of patients with depressive disorders ${ }^{19}$.
The present study showed that $12.3 \%$ of the general hospitals surveyed did not provide psychiatry clinics and wards. Therefore, there is a need to strengthen the provision of psychiatric services in general hospitals. Furthermore, the analysis revealed a lack of specialized medical staff in the general hospitals surveyed, with only a median of six psychiatric clinicians in each hospital. It is likely that such a small number of psychiatric clinicians per hospital would struggle to meet the workload, particularly as this appears to be increasing. Therefore, we believe it will be necessary to increase the number of psychiatric clinicians in general hospitals. Similarly, mental health nurses are also subjected to high work-related stress ${ }^{20}$, including family-related stress and workplace violence ${ }^{20,21}$. One of the most important risk factors for work-related stress is a shortage of staff allocation ${ }^{21}$.

Guidelines for training in consultation-liaison psychiatry and psychosomatics have been developed in Europe ${ }^{22}$, but such guidelines are not available in China. In the present study, it was found that only 19 hospitals had conducted psychiatry-related training over the 3-year period, highlighting an important deficiency. Additionally, the respondents considered it essential to set up psychiatry clinics and wards in general hospitals to enhance the rate of psychiatry consultations and promote scientific research, with the aim of improving the diagnosis and treatment of mental health disorders. A study from Belgium reported that the practice environment is directly impacted by staff job satisfaction, intention to stay, and the perception of the quality of care $^{23}$. Compared with Western countries, 
these aspects are not sufficiently enforced in China, especially job satisfaction, which is generally low in China ${ }^{24,25}$, as well as training ${ }^{26}$.

The present study showed that only a small proportion (6.9\%) of the non-psychiatric staff in general hospitals had sufficient knowledge of and training in the manifestations of mental health disorders. In addition, only a small proportion of the respondents understood the diagnostic criteria (4.5\%) and treatment protocols $(3.8 \%)$. This underlines the need for further training in order to detect the symptoms of mental health disorders in non-psychiatric departments. Similar trends were observed in Hunan (China) ${ }^{13}$, Taiwan ${ }^{27}$, Australia $^{28}$, the United Kingdom ${ }^{29}$, India ${ }^{30}$, Sweden ${ }^{31}$ and Kenya ${ }^{32}$, among others. Since this important issue was first documented as early as the $1990 \mathrm{~s}^{12}$, it seems that not enough has been done to address this shortcoming and that additional efforts are needed.

This study's main strengths include its large number of respondents and high rates of response. Nevertheless, this study has some limitations. Firstly, although different levels of hospitals were included in the survey, the selection was not based on random sampling, which may have introduced bias. However, the selection of four provinces in different regions of China (central, eastern, northern and southern) increases the likelihood that the findings are generalizable to China as a whole. Secondly, the survey of the 1,152 clinicians was conducted using a questionnaire that was not validated, and this also may have introduced bias. Thirdly, factors that might be associated with mental healthcare resources (e.g. hospital grade) or clinician knowledge of anxiety and depressive disorders (e.g. age or clinical specialty) were not investigated.

In conclusion, assessment of the psychiatric care resources of 57 general hospitals in four provinces of China over a 3-year period showed that clinicians and nurses were severely lacking in psychiatryrelated training. We conclude that there is an urgent need to set up psychiatry/psychology wards and/or clinics in general hospitals and strengthen psychiatric resources and training in order to improve the provision of mental health care in general hospitals in China.

\section{Acknowledgments}

The authors would like to express their gratitude to all the study participants.

\section{Funding}

This study was supported by the National Key Technology Research and Development Program of the Ministry of Science and Technology of China (2012BAI01B05) and the National Natural Science Foundation of China (81201058, 30971040).

\section{Conflict of interests}

All authors declare that they have no any conflict of interests.

\section{References}

1. Demyttenaere K, Bruffaerts R, Posada-Villa J, Gasquet I, Kovess V, Lepine JP, et al. Prevalence, severity, and unmet need for treatment of mental disorders in the World Health Organization World Mental Health Surveys. JAMA. 2004;291(21):2581-90.

2. Phillips MR, Zhang J, Shi Q, Song Z, Ding Z, Pang S, et al. Prevalence, treatment, and associated disability of mental disorders in four provinces in China during 2001-05: an epidemiological survey. Lancet. 2009;373(9680):2041-53.

3. Townsend M. Psychiatric Mental Health Nursing: Concepts of Care in Evidence-Based Practice. Philadelphia: F.A. Davis Company; 2015.

4. Celano CM, Suarez L, Mastromauro C, Januzzi JL, Huffman JC. Feasibility and utility of screening for depression and anxiety disorders in patients with cardiovascular disease. Circ Cardiovasc Qual Outcomes. 2013;6(4):498-504.

5. Tu HP, Lin CH, Hsieh HM, Jiang HJ, Wang PW, Huang CJ. Prevalence of anxiety disorder in patients with type 2 diabetes: a nationwide population-based study in Taiwan 2000-2010. Psychiatr Q. 2017;88(1):75-91.
6. Anderson C, Tauch D, Quante A. Diagnosis and Treatment Procedures for Patients With Anxiety Disorders by the Psychiatric Consultation Liaison Service in a General Hospital in Germany: A Retrospective Analysis. Prim Care Companion CNS Disord. 2015;17(5):10.4088/PCC.15m01805.

7. Qin X, Wang W, Jin Q, Ai L, Li Y, Dong G, et al. Prevalence and rates of recognition of depressive disorders in internal medicine outpatient departments of 23 general hospitals in Shenyang, China. J Affect Disord. 2008;110(1-2):46-54.

8. Rentsch D, Dumont P, Borgacci S, Carballeira Y, deTonnac N, Archinard $\mathrm{M}$, et al. Prevalence and treatment of depression in a hospital department of internal medicine. Gen Hosp Psychiatry. 2007;29(1):25-31.

9. Qin X, Phillips MR, Wang W, Li Y, Jin Q, Ai L, et al. Prevalence and rates of recognition of anxiety disorders in internal medicine outpatient departments of 23 general hospitals in Shenyang, China. Gen Hosp Psychiatry. 2010;32(2):192-200.

10. Zhong BL, Chen HH, Zhang JF, Xu HM, Zhou C, Yang F, et al. Prevalence, correlates and recognition of depression among inpatients of general hospitals in Wuhan, China. Gen Hosp Psychiatry. 2010;32(3):268-75.

11. Yan ZY, Gu MJ, Zhong BL, Wang C, Tang HL, Ling YQ, et al. Prevalence, risk factors and recognition rates of depressive disorders among inpatients of tertiary general hospitals in Shanghai, China. J Psychosom Res. 2013;75(1):65-71.

12. Keshavan K, Sriram TG, Kaliaperumal VG, Subramanya KR. Mental health knowledge and skills of general hospital nursing staff: an exploratory study. Int J Soc Psychiatry. 1991;37(4):280-4

13. Wu Q, Luo X, Chen S, Qi C, Long J, Xiong Y, et al. Mental health literacy survey of non-mental health professionals in six general hospitals in Hunan Province of China. PLoS One. 2017;12(7):e0180327.

14. Al-Huthail YR. Accuracy of referring psychiatric diagnosis. Int $J$ Health Sci (Qassim). 2008;2(1):35-8.

15. Liu C, Chen L, Xie B, Yan J, Jin T, Wu Z. Number and characteristics of medical professionals working in Chinese mental health facilities. Shanghai Arch Psychiatry. 2013;25(5):277-85.

16. Zhong Q, Yan H, Wu R, Tan Y, Tang H. China Mental Health Reform perspective about psychiatric nursing assistants. J Prev Med Healthcare. 2017;1:1002.

17. Yu C, Lyu J, Chen Y, Guo Y, Paul S, Bian Z, et al. [Epidemiology of major depressive episodes among Chinese adults aged 30-79 years: data from the China Kadoorie Biobank]. Zhonghua Liu Xing Bing Xue Za Zhi. 2015;36(1):52-6.

18. Wang L, Song R, Chen Z, Wang J, Ling F. Prevalence of depressive symptoms and factors associated with it in type 2 diabetic patients: a cross-sectional study in China. BMC Public Health. 2015;15:188.

19. Liu C, Liu M, Jiang R, Ma H, Wu X, Luan S, et al. Prevalence and Recognition of Depressive Disorder in Three Medical Outpatient Departments of General Hospitals in Beijing, China. J Nerv Ment Dis. 2016;204(7):537-41.

20. Zeng JY, An FR, Xiang YT, Qi YK, Ungvari GS, Newhouse R, et al. Frequency and risk factors of workplace violence on psychiatric nurses and its impact on their quality of life in China. Psychiatry Res. 2013;210(2):510-4.

21. Yao X, Li Z, Arthur D, Hu L, Cheng G. The application of a violence risk assessment tool among Chinese psychiatric service users: a preliminary study. J Psychiatr Ment Health Nurs. 2012;19(5):438-45.

22. Sollner W, Creed F; European Association of Consultation-Liaison Psychiatry and Psychosomatics Workgroup on Training in ConsultationLiaison. European guidelines for training in consultation-liaison psychiatry and psychosomatics: report of the EACLPP Workgroup on Training in Consultation-Liaison Psychiatry and Psychosomatics. J Psychosom Res. 2007;62(4):501-9.

23. Van Bogaert P, Wouters K, Willems R, Mondelaers M, Clarke S. Work engagement supports nurse workforce stability and quality of care: nursing team-level analysis in psychiatric hospitals. J Psychiatr Ment Health Nurs. 2013;20(8):679-86.

24. Lu Y, Hu XM, Huang XL, Zhuang XD, Guo P, Feng LF, et al. Job satisfaction and associated factors among healthcare staff: a cross-sectional study in Guangdong Province, China. BMJ Open. 2016;6(7):e011388.

25. Liu J, Yu W, Ding T, Li M, Zhang L. Cross-sectional survey on job satisfaction and its associated factors among doctors in tertiary public hospitals in Shanghai, China. BMJ Open. 2019;9(3):e023823.

26. Li J, Li J, Thornicroft G, Yang H, Chen W, Huang Y. Training community mental health staff in Guangzhou, China: evaluation of the effect of a new training model. BMC Psychiatry. 2015;15:263. 
27. Liu SI, Lu RB, Lee MB. Non-psychiatric physicians' knowledge, attitudes and behavior toward depression. J Formos Med Assoc. 2008;107(12):921-31.

28. Reed F, Fitzgerald L. The mixed attitudes of nurse's to caring for people with mental illness in a rural general hospital. Int J Ment Health Nurs. 2005;14(4):249-57.

29. Rayner L, Matcham F, Hutton J, Stringer C, Dobson J, Steer S, et al. Embedding integrated mental health assessment and management in general hospital settings: feasibility, acceptability and the prevalence of common mental disorder. Gen Hosp Psychiatry. 2014;36(3):318-24.
30. Pande V, Saini R, Chaudhury S. Attitude toward mental illness amongst urban nonpsychiatric health professionals. Ind Psychiatry J. 2011;20(1):17-20.

31. Mårtensson G, Jacobsson JW, Engström M. Mental health nursing staff's attitudes towards mental illness: an analysis of related factors. J Psychiatr Ment Health Nurs. 2014;21(9):782-8.

32. Ndetei DM, Khasakhala LI, Mutiso V, Mbwayo AW. Knowledge, attitude and practice (KAP) of mental illness among staff in general medical facilities in Kenya: practice and policy implications. Afr J Psychiatry (Johannesbg). 2011;14(3):225-35. 